Old Dominion University

ODU Digital Commons

VMASC Publications

Virginia Modeling, Analysis \& Simulation Center

2013

\title{
Path Clearance for Emergency Vehicles Through the Use of Vehicle-to-Vehicle Communication
}

\author{
Craig Jordan \\ Old Dominion University, cajordan@odu.edu \\ Mecit Cetin \\ Old Dominion University, mcetin@odu.edu \\ R. Michael Robinson \\ Old Dominion University, rmrobins@odu.edu
}

Follow this and additional works at: https://digitalcommons.odu.edu/vmasc_pubs Part of the Civil Engineering Commons, Transportation Commons, and the Transportation
Engineering Commons

\section{Repository Citation}

Jordan, Craig; Cetin, Mecit; and Robinson, R. Michael, "Path Clearance for Emergency Vehicles Through the Use of Vehicle-toVehicle Communication" (2013). VMASC Publications. 26.

https://digitalcommons.odu.edu/vmasc_pubs/26

\section{Original Publication Citation}

Jordan, C. A., Cetin, M., \& Robinson, R. M. (2013). Path clearance for emergency vehicles through the use of vehicle-to-vehicle communication. Transportation Research Record, 2381, 45-53. doi:10.3141/2381-06

This Article is brought to you for free and open access by the Virginia Modeling, Analysis \& Simulation Center at ODU Digital Commons. It has been accepted for inclusion in VMASC Publications by an authorized administrator of ODU Digital Commons. For more information, please contact digitalcommons@odu.edu. 


\title{
Path Clearance for Emergency Vehicles Through the Use of Vehicle-to-Vehicle Communication
}

\author{
Craig A. Jordan, Mecit Cetin, and R. Michael Robinson
}

\begin{abstract}
The study described in this paper evaluated and tested a new strategy to enable emergency response vehicles (EVs) to navigate through congestion at signalized intersections more efficiently. The proposed strategy involves the use of vehicle-to-vehicle communication to send messages to alert vehicles to the approach of the $\mathrm{EV}$ and to provide specific instructions on maneuvering to allow the EV to proceed through congested signalized intersections as quickly as possible. This movement is achieved by creation of a split in the vehicle queue in one lane at a critical location to allow the $\mathrm{EV}$ to proceed at its desired speed but minimize the disruption to the rest of the traffic. The proposed method uses kinematic wave theory (i.e., shock wave theory) to determine the critical point in the vehicle queue. The proposed method is simulated in a microscopic traffic simulator for evaluation. The results show that this strategy can significantly shorten the travel time for $\mathrm{EVs}$ s through congested signalized intersections.
\end{abstract}

Vehicle-to-vehicle (VTV) and vehicle-to-infrastructure (VTI) communications are being used to develop new applications to improve system operations and safety. Through the sharing of vehicle information, such as speed and location, between vehicles and the infrastructure, a more efficient transportation network can be created. Some areas of active research include improved on-ramp merging at freeways ( 1 ), cooperative driving (2), intelligent and safer signal timing design and control $(3,4)$, queue length estimation $(5,6)$, and travel time estimation across transportation networks to develop real-time route guidance and traveler information systems (7). The application of these systems has the potential to provide travelers with detailed information on the status of the transportation network.

An area that has seen an increase in research pertains to emergency response vehicles (EVs), including police vehicles, ambulances, and fire trucks. An EV equipped with a VTV or a VTI communication system could have improve response times by transmitting the location, route, and final destination to vehicles and infrastructure in its path (8). ERTICO, an intelligent transportation system firm in Europe, has developed a rescue system (http://www.ertico.com/ assets/download/GST/RESCUE.pdf) that allows vehicles to be outfitted with a communication device and visual display to alert drivers

C. A. Jordan and R. M. Robinson, Virginia Modeling Analysis and Simulation Center, Old Dominion University, 1030 University Boulevard, Suffolk, VA 23435. M. Cetin, Department of Civil and Environmental Engineering, Old Dominion University, Kaufman Hall 135, Norfolk, VA 23529-0241. Corresponding author: C. A. Jordan, cajordan@odu.edu.

Transportation Research Record: Journal of the Transportation Research Board, No. 2381, Transportation Research Board of the National Academies, Washington, D.C., 2013, pp. 45-53.

DOI: $10.3141 / 2381-06$ to the approach of an EV. The system also allows the transmission of data between equipped non-EVs to alert drivers to the location of the EV at the emergency scene.

Provision of the best possible route for an EV depends on the geometric features of the roadway network as well as the traffic conditions. Under light traffic on multilane highways with wide lanes or shoulders, vehicles can be alerted to the approach of an EV and clear a particular lane to allow the EV to pass unobstructed. However, on narrow roadways under congested conditions, no solution that would allow an EV to travel at its desired speed through the intersection is obvious.

The study described in this paper evaluated a strategy to allow EVs to travel congested roadway segments that have signalized intersections. In particular, the paper addresses EVs in queues on two-lane divided roadways without shoulders at traffic signals. The objective is to manage the queued traffic so that the EV clears the intersection safely and as quickly as possible but minimize the impacts on other traffic. The evaluation of the strategy was performed in a microscopic traffic simulator, which provides flexibility in testing environments and duplication of traffic patterns for comparison of strategies. The exchange of information between vehicles was not explicitly modeled. It was assumed that all vehicles can receive the messages sent by the $\mathrm{EV}$ and comply with the given instructions.

The strategy developed includes stopping of traffic on one lane at a critical point to allow the EV to change lanes so that it can travel unimpeded through the intersection. The critical point is found by use of the Lighthill-Whitham-Richards (LWR) model (9). The LWR model is a linear model used to describe traffic flow dynamics and is well suited for prediction of shock waves. It arises from the principle of the conservation of vehicles and a fundamental diagram that relates flow to density.

The paper demonstrates how the LWR model can be used to make predictions about the evolution of traffic over time and space and how such information can be used to alter vehicle trajectories to improve EV travel times under congested conditions.

\section{BACKGROUND AND LITERATURE REVIEW}

EVs are painted with specific colors and are equipped with audible and visual devices for identification and to alert other vehicles to their relative position. The devices and markings communicate to drivers that an EV is near, prompting them to respond according to state guidelines. Communication effectiveness is limited by background and in-vehicle noise and is dependent on whether vehicles are within visual and audible range. Drivers can have difficulty identifying the specific location of the source and the path of the EV (10). 
In addition to acknowledging the presence of an $\mathrm{EV}$, drivers need to know where the EV is and how to react appropriately to its approach. State guidelines instruct drivers to change lanes to the right, if applicable, and to stop when an EV approaches from behind. Drivers must know the location of the EV, the direction in which the EV is going, and what they should do to allow the EV to pass safely. The lack of understanding on where to go or where the EV is located has been identified as a cause of EV crashes. Auerbach et al. reported that drivers who were involved in a collision with an ambulance frequently stated that they were unaware of the ambulance's presence (11). A separate study on ambulance crash data reported that in 2009, the United States had a total of 1,404 ambulance crashes while the ambulance was using lights and sirens. The report was based on data from the Fatality Analysis Reporting System, NHTSA, and the General Estimates System of the National Automotive Sampling System (12).

In the majority of collisions that involve an $\mathrm{EV}$, the $\mathrm{EV}$ does not continue on the emergency call (13). Another EV needs to be dispatched to the original call. The result is a significant delay before aid can be provided. Auerbach et al. reported that when an ambulance is involved in a collision, an average delay of 9.4 min results before the original patient reaches the hospital (11). In addition to the response delay, an EV often needs to be dispatched to the collision location involving the original $\mathrm{EV}$.

A study conducted in the United Kingdom indicated that a reduction in the amount of time required to respond to people in need of emergency cardiac care has the potential to increase the rate of patient survival (14). The study indicated that a reduction in the amount of time required to respond to cardiac patients from 14 to 8 min could increase the proportion of survivors from $6 \%$ to $8 \%$ and a reduction to 5 min could increase the proportion of survivors by between $10 \%$ and $11 \%$.

The American Heart Association also emphasizes the importance of early response. It reports that for each minute between the time of a cardiac arrest and the time that a defibrillator is used, the survival rate is reduced by between $7 \%$ and $10 \%$ (15).

Studies of EVs that have been performed have not specifically addressed strategies to aid EVs through signalized intersections. Moussa developed a lane-changing strategy that focused on the evacuation of EVs on highways (16). The strategy involved the creation of gaps between vehicles on a two-lane roadway through the sending of messages that instruct non-EVs to change lanes to the higher-density lane.
Toy et al. used unique strategies to assist EVs as they travel to destinations through congestion on highways (17). They evaluated the manipulation of vehicles on an automated highway to aid with the advancement of an EV as quickly as possible. The strategies involved the grouping of vehicles into platoons and shifting of the vehicles left, right, forward, and backward to form gaps on the congested highway segment to allow the EV to pass.

Yoo et al. developed a strategy to reduce response times by reserving lanes on roadway segments along an EV's route (18). Non-EVs are instructed to move out of the reserved lane to provide a path for the $\mathrm{EV}$. The study introduced a short-range implementation (when the EV encounters vehicles) and a long-range implementation (over the entire EV route) to assist with the response time. This study did not address traffic congestion or how to handle vehicles that cannot change lanes because of the volume of traffic.

\section{SOLUTION FORMULATION}

As a traffic light alternates between green and red phases, it creates discontinuities, or shock waves, in the traffic stream. Use of the LWR theory is a particularly suitable way to predict these shock waves because the boundary conditions are well-defined (e.g., the backward-moving shock waves start at the stop bar when the signal phase changes). Furthermore, the queue-discharging process at signalized intersections has been shown to be quite stable, which enables reliable prediction of the speed of the shock wave (19).

Figure 1 shows a diagram of a typical shock wave for the formation and dissipation of a queue at a traffic light. Backward-moving shock waves start at the beginning of the red phase $\left(t_{R}\right)$ and the time when the EV joins the back of the queue and the traffic signal turns green $\left(t_{0}\right)$ and thus represent the back of the queue and the front of the queue (or discharging process), respectively. The speed of the shock wave for the queue discharge $(w)$ and the free-flow speeds (or desired speeds) of regular vehicles and the EV are assumed to be known.

\section{Clearance of Path for EV Through One Intersection}

A strategy involving clearance of a path for an EV through one signalized intersection on a two-lane congested roadway facility as quickly as possible with minimal impact to the background traffic

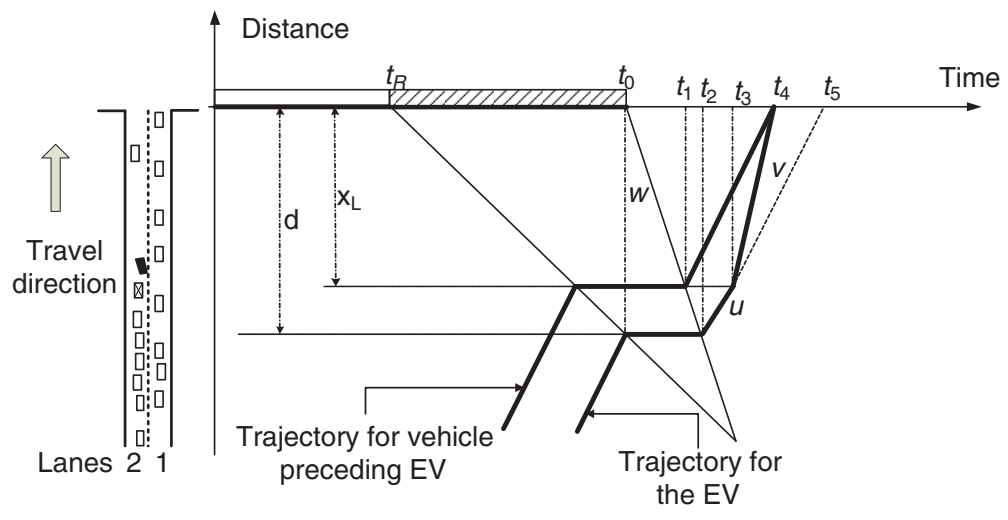

FIGURE 1 Shock wave profile for single queue at traffic light and trajectories of $E V$ and preceding vehicle. 
is described. The EV is in a vehicle queue waiting for the signalized intersection to turn green and receives a call to travel to an arbitrary point downstream of the intersection.

The solution to this problem was developed through the use of shock wave analysis and involves stopping of vehicles in the adjacent lane to clear a path for the EV to change lanes and travel unimpeded through the signalized intersection. Figure 1 provides a shock wave profile of this strategy. The EV is represented by the black vehicle on the left side of Figure 1 and initially starts in Lane 1. Its trajectory is represented by the thick back line.

At time $t_{0}$, the $\mathrm{EV}$ receives a call instructing it to proceed to a destination downstream of the signalized intersection. Immediately after it receives the call, the EV sends a message to the traffic signal to turn green and to the vehicle located at distance $x_{L}$ from the stop bar (the vehicle denoted with an X in Figure 1) to hold its position. At time $t_{1}$, the departure shock wave reaches the vehicle preceding the stopped vehicle. The preceding vehicle departs and a gap forms between it and the stopped vehicle. At time $t_{2}$, the departure shock wave reaches the $\mathrm{EV}$, which allows it to proceed forward but with an initial velocity that is the desired speed of the background traffic $(u)$. When the EV passes the stopped vehicle located at $x_{L}$ from the stop bar (time $t_{3}$ ), the EV changes lanes and travels at a velocity that is the desired speed of the EV (v) through the intersection. The trajectories of the EV and the vehicle preceding the stopped vehicle meet at time $t_{4}$.

If it is assumed that the location of the EV in the vehicle queue and the velocities of the EV and the background vehicles are known, a formulation based on the shock waves can be developed to determine the critical location at which a vehicle in the adjacent lane may be stopped for a short duration to make way for the EV. The formulation is described below, and the following variables are used in the formulation:

$$
\begin{aligned}
w= & \text { speed of shock wave for discharging flow at signalized inter- } \\
& \text { section, } \\
v= & \text { desired speed of EV, } \\
u= & \text { desired speed of background vehicles, } \\
x_{L}= & \text { critical distance from intersection to point where queue needs } \\
& \text { to be split, and } \\
d= & \text { distance from EV (when in queue) to intersection. }
\end{aligned}
$$

If it is assumed that the time when the EV receives the message $\left(t_{0}\right)$ is 0 , the time at which the vehicle preceding the stopped vehicle departs the queue $\left(t_{1}\right)$ can be found by division of the distance $x_{L}$ by the shock wave departure speed $(w)$. The equation is as follows:

$t_{1}=\frac{x_{L}}{w}$

The departure time for the EV from the queue (time $t_{2}$ ) can also be found by division of its distance from the intersection $(d)$ by the shock wave speed $w$ :

$t_{2}=\frac{d}{w}$

The time when the EV changes lanes at point $x_{L}$ (time $t_{3}$ ) and begins traveling at its desired speed can be found by division of the distance between the EV and the stopped vehicle by the initial speed $(u)$ and addition of the value to time $t_{2}$.
$t_{3}=t_{2}+\frac{d-x_{L}}{u}=\frac{d}{w}+\frac{d-x_{L}}{u}$

After the EV changes lanes, it travels to the intersection, reaching it at the same time as the preceding vehicle (time $t_{4}$ ). The time can be calculated for both the EV and the preceding vehicle with two equations. For the preceding vehicle, the equation is

$t_{4}=t_{1}+\frac{x_{L}}{u}=\frac{x_{L}}{w}+\frac{x_{L}}{u}$

For the $\mathrm{EV}$, the equation is

$t_{4}=t_{3}+\frac{x_{L}}{v}=\frac{d}{w}+\frac{d-x_{L}}{u}+\frac{x_{L}}{v}$

The simultaneous solution of Equations 4 and 5 for $x_{L}$ results in the following relationship:

$x_{L}=d \frac{w^{-1}+u^{-1}}{w^{-1}+2 u^{-1}-v^{-1}}$

If the queue on Lane 2 is split at location $x_{L}$, the EV will be able to travel over distance $x_{L}$ at its desired speed $(v)$. Travel at this speed will result in a theoretical time savings that is equal to the difference $t_{5}-t_{4}$.

\section{Clearance of Path for EV for Two Intersections}

In a scenario involving clearance of a path for an EV for two intersections, the destination of the EV is located at a point beyond two intersections. The shock wave corresponding to this scenario is shown in Figure 2. The trajectories of the EV and the preceding vehicle are shown as described for Figure 1. The idea is to find the critical point at which traffic in the adjacent lane should be stopped so the EV can change lanes to travel at its desired speed (v) through the upstream intersection and travel unimpeded through the downstream intersection. For this scenario to be successful, traffic signal preemption needs to occur at the upstream and downstream intersections.

To formulate the critical point at which the platoon should be split, limiting assumptions need to be made. The downstream vehicle queue is assumed to be moving before the first vehicle in the upstream platoon reaches the back of the queue. In addition, no traffic from access points other than the first intersection is considered. The speeds of the discharging shock wave from the upstream and downstream intersections are assumed to be equal. Additionally, it is assumed that sufficient storage exists downstream of the intersections to accept the discharging vehicles.

The formulation for calculation of the critical point at which the platoon should be split is similar to that in the previous scenario, but with the inclusion of a new variable, the distance between intersections $(z)$. The formulation for the timing of the preemption is provided after the platoon-split formulation.

If it is assumed that $t_{0}$ is 0 , the departure time from the queue for the preceding vehicle $\left(t_{1}\right)$ can be found as follows:

$t_{1}=\frac{x_{L}}{w}$ 


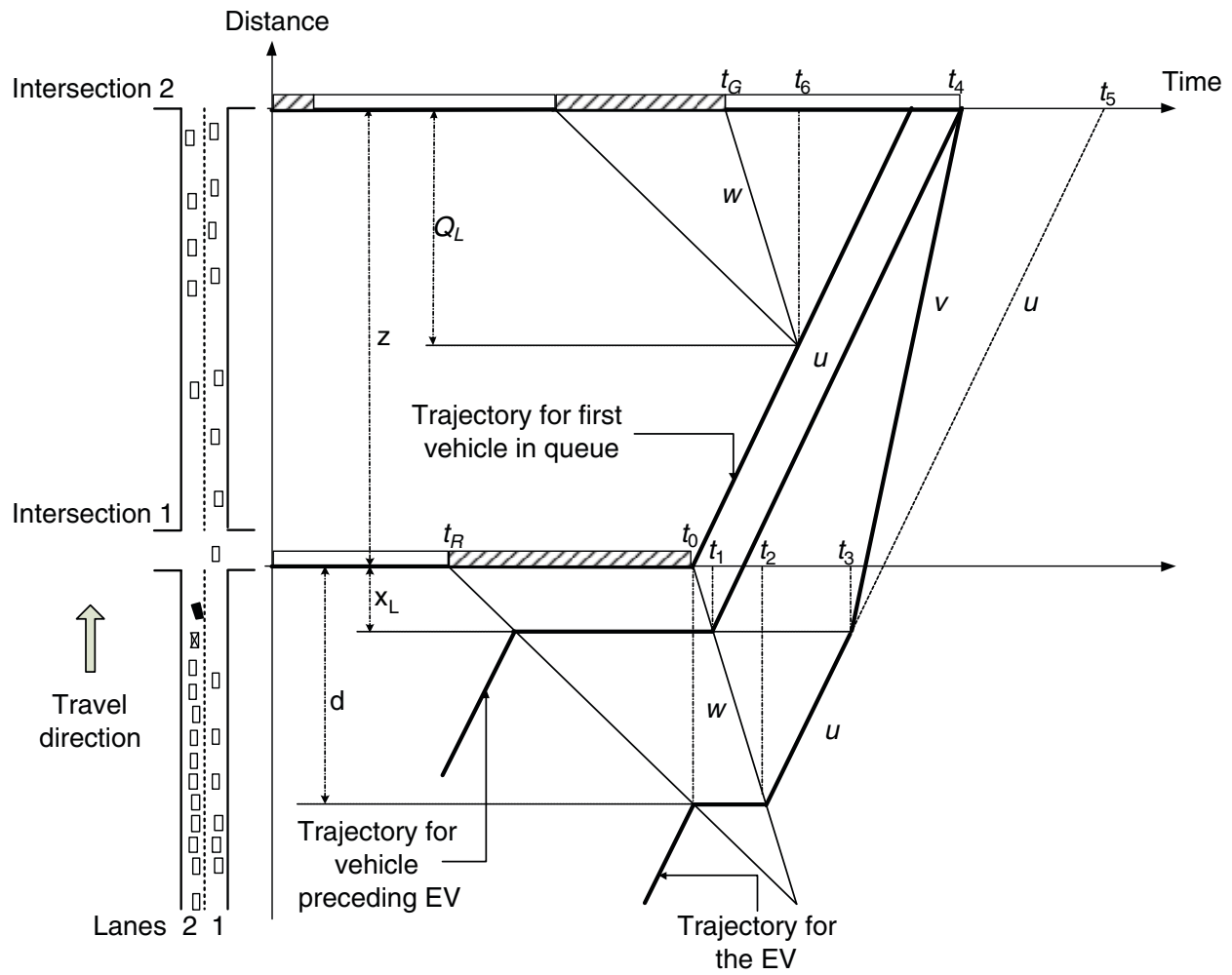

FIGURE 2 Shock wave diagram for single queue at traffic light and trajectories of EV and preceding vehicle to downstream intersection.

The departure time from the queue for the $\operatorname{EV}\left(t_{2}\right)$ is

$t_{2}=\frac{d}{w}$

The time when the EV changes lanes and starts traveling at its desired speed $\left(t_{3}\right)$ can be found as

$t_{3}=t_{2}+\frac{d-x_{L}}{u}=\frac{d}{w}+\frac{d-x_{L}}{u}$

The time at which the vehicle preceding the EV and the EV reach the downstream intersection at the same time $\left(t_{4}\right)$ is calculated with the following two equations, respectively:

$t_{4}=t_{1}+\frac{x_{L}+z}{u}=\frac{x_{L}}{w}+\frac{x_{L}+z}{u}$

$t_{4}=t_{3}+\frac{x_{L}+z}{v}=\frac{d}{w}+\frac{d-x_{L}}{u}+\frac{x_{L}+z}{v}$

The simultaneous solution of Equations 10 and 11 for $x_{L}$ provides the following equation:

$x_{L}=\frac{d\left(w^{-1}+u^{-1}\right)+z\left(v^{-1}-u^{-1}\right)}{\left(w^{-1}+2 u^{-1}-v^{-1}\right)}$

If the queue in Lane 2 is split at location $x_{L}$, the EV will be able to travel over the distance $x_{L}+z$ at its desired speed $(v)$. The result will be time savings that equals the difference $t_{5}-t_{4}$.
To clear potential vehicle queues at the downstream intersection, a formulation was developed by use of the LWR method to specify the time at which the downstream intersection turns green in reference to when the EV enters the back of the queue (time $t_{0}$ ). Figure 2 shows the shock waves that define the boundaries of the queue and the trajectory of the EV. The formulation for calculation of this critical time is provided below and uses a new variable, the length of the queue at the downstream intersection $\left(Q_{L}\right)$.

The time that the first vehicle departing from the upstream intersection reaches the back of the queue at the downstream intersection $\left(t_{6}\right)$ can be found as

$t_{6}=\frac{z-Q_{L}}{u}$

The time that it takes for the vehicle queue at the downstream intersection to discharge can be calculated with

$t_{6}=t_{G}+\frac{Q_{L}}{w}$

where $t_{G}$ is the time that the signal turns green.

The simultaneous solution of Equations 13 and 14 for $t_{G}$ provides the following equation:

$t_{G}=\frac{z-Q_{L}}{u}-\frac{Q_{L}}{w}$

Time $t_{G}$ is relative to time $t_{0}$; therefore, the downstream intersection should turn green $t_{G}$ seconds after time $t_{0}$. 
The formulation developed in this paper assumes a simplified transportation system between two intersections and provides a straightforward solution for the platoon-split strategy. Although the scenario for two intersections is simplified, it is still complex, and the equation that determines the platoon-split location $\left(x_{L}\right)$ is bounded by certain conditions. The following paragraphs provide a discussion on the limitations of Equation 12.

The first limitation involves the length of the queue at the downstream intersection $\left(Q_{L}\right)$. If the traffic signal at this intersection is red, the earliest that it can turn green is at time $t_{0}$ (when the EV receives the call from dispatch). If the vehicle queue is longer than a certain length, the vehicles discharging from the upstream intersection and the EV will have to slow down before the downstream intersection (Figure 3). The maximum length that the downstream queue $\left(Q_{L}\right)$ can be to provide enough time for the EV to travel through the downstream intersection unimpeded is formulated as follows.

Equation 13 provides the time that it takes for the first vehicle at the upstream intersection to reach the back of the downstream intersection queue. If it is assumed that the upstream and downstream traffic signals turn green at time $t_{0}$, the time that the last vehicle in the downstream queue starts to move is found with the following equation:

$t_{6}=\frac{Q_{L}}{w}$

The simultaneous solution of Equations 13 and 16 for $Q_{L}$ shows that the maximum length that the downstream vehicle queue can be for the scenario equation to hold $\left(Q_{L_{\max }}\right)$ is as follows:

$Q_{L_{\max }}=z \frac{u^{-1}}{w^{-1}+u^{-1}}$
The second limitation is related to the location of the EV in the vehicle queue at the upstream intersection. If the location of the EV is close to the intersection, the solution (Equation 6) will provide a platoon-split location that is downstream of the upstream intersection. This will allow the EV to change lanes before the critical location and catch the preceding vehicle before the downstream intersection (Figure 3). To determine the minimum value for $d\left(d_{\min }\right)$, the value of $x_{L}$ is set equal to 0 in Equation 12. Solution of the equation for $d$ produces the following equation:

$d_{\min }=z \frac{u^{-1}-v^{-1}}{w^{-1}+u^{-1}}$

\section{SIMULATION}

The VISSIM microscopic traffic simulation package of the PTV Group was used to evaluate the formulated algorithms. VISSIM applies discrete time- and agent-based simulation to model traffic operations. Each vehicle is simulated as a separate object with a specific set of car-following and lane-changing behaviors. The program tracks specific attributes, such as speed, location, and vehicle type, for every vehicle at each time step. The unique driving characteristics and flexibility of the software allow complex transportation roadway networks to be developed.

VISSIM provides users the ability to control certain functions and attributes of the microscopic simulation with outside programs during the simulation runs through the use of the component object model $(\mathrm{COM})$ interface. This allows information to be passed between the programs for implementation of the study algorithms. The COM interface was used to track the location of vehicles, calculate $x_{L}$, instruct

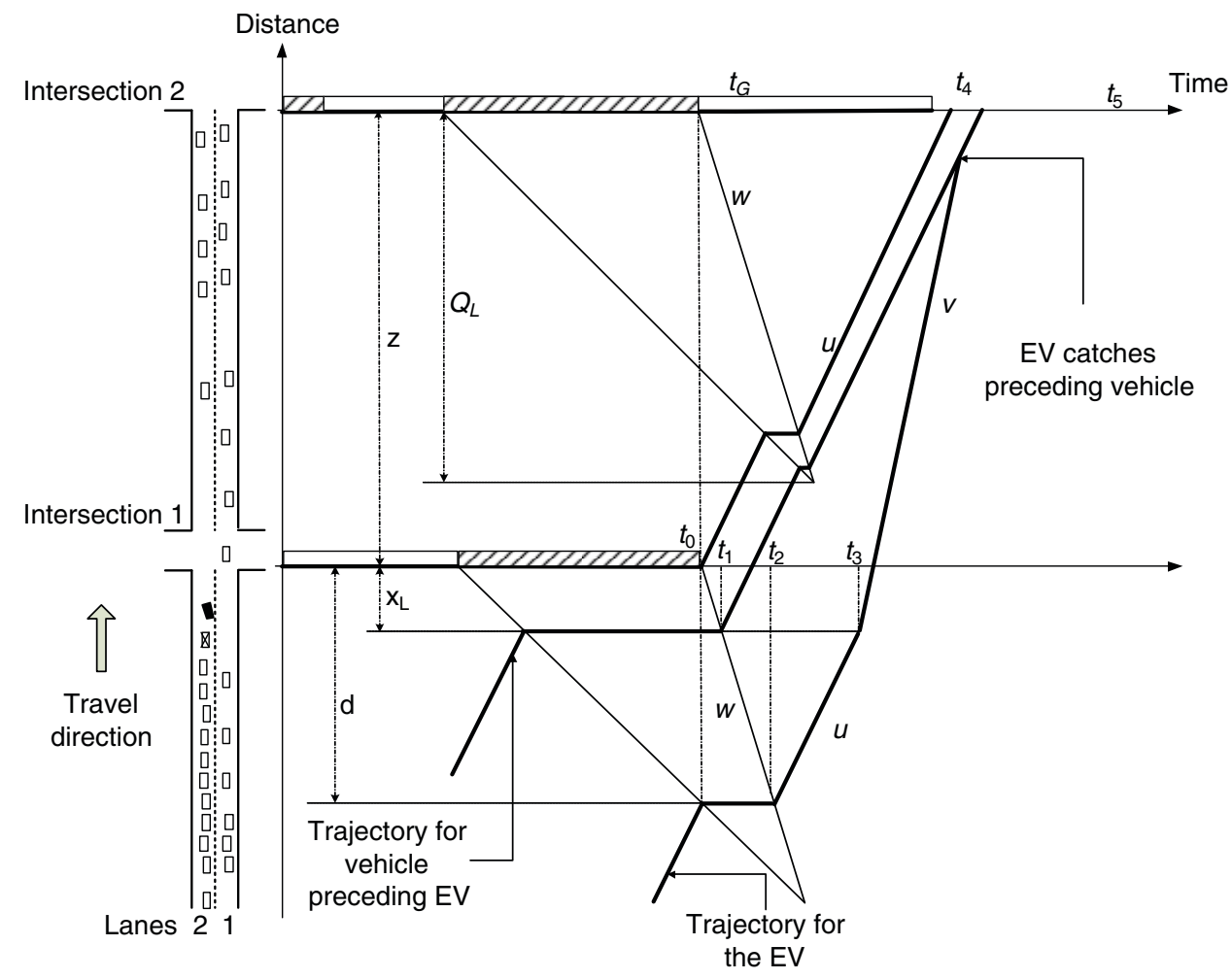

FIGURE 3 Shock wave diagram for boundary condition $Q_{L}$. 
vehicles to stop upstream of $x_{L}$, initiate lane changing of the $\mathrm{EV}$, and change the signal phases.

The roadway network used in the simulation consisted of a straight two-lane roadway approximately $2 \mathrm{~km}$ in length with two intersections and no other obstructions. Traffic was loaded at a rate of 800 vehicles per hour per lane to create traffic congestion at the signalized intersections. The traffic composition consisted of passenger cars only to provide similar driving behaviors. Simulation runs were performed with a set of random seeds, and EVs were added to the network at different random times to produce various levels of traffic congestion and vehicle queues of various lengths of at the intersections.

\section{Simulation Scenarios}

To determine if the strategies developed would work in a simulated environment, six scenarios were run for comparison and are listed below:

- One intersection:

-Only signal preemption (the EV in the vehicle queue does not have equipment to alert drivers to its presence) and

-Platoon-split strategy with preemption and

- Two intersections:

-Only signal preemption,

$-\mathrm{EV}$ with siren (the $\mathrm{EV}$ in the vehicle queue has a siren to alert drivers of its presence),

$-\mathrm{EV}$ with siren and preemption (the EV in the vehicle queue has a siren and the traffic signal is equipped with preemption), and

-Platoon-split strategy with preemption.

The platoon-split simulation runs were performed for each of the following vehicle speeds, which reflect a sample of speeds that can be found in urban environments:

Speed Setting 1. Non-EVs at $50 \mathrm{~km} / \mathrm{h}$ and the EV at 80 at $\mathrm{km} / \mathrm{h}$, Speed Setting 2. Non-EVs at $50 \mathrm{~km} / \mathrm{h}$ and the EV 65 at $\mathrm{km} / \mathrm{h}$, and

Speed Setting 3. Non-EVs at $72 \mathrm{~km} / \mathrm{h}$ and the EV at $86 \mathrm{~km} / \mathrm{h}$.

Because of time constraints, the simulation run with the EV with a siren and the simulation run with the EV with a siren and the traffic signal equipped with preemption were performed with Speed Setting 1 only. In addition, the scenarios with two intersections included the evaluation of three intersection spacing values $(1,000$, 500 , and $250 \mathrm{~m}$ ).

\section{Assumptions and Limitations}

The following is a list of assumptions that were used in the simulation runs:

1. The roadway network is equipped with VTI communication systems.

2. All vehicles are equipped with VTV and VTI communication systems and comply with the given instructions.

3. The information exchanged between vehicles and the infrastructure has a range of $300 \mathrm{~m}$ and occurs without delay or failure.
4. All non-EVs have the same desired speed.

5. Non-EVs can change lanes only when EVs approach from behind.

\section{RESULTS}

This section provides the results of the simulation runs for the scenarios identified in the previous sections. Analyses were performed to compare the travel time from each simulation run with that under the base conditions.

\section{Theoretical Results}

The theoretical travel time improvements for the scenario with one intersection can be determined from Figure 1 by comparison of the arrival time of the EV with and without the use of the platoonsplit strategy (time $t_{4}$ and time $t_{5}$, respectively). The dotted line from location $x_{L}$ to time $t_{5}$ in Figure 1 illustrates the EV trajectory without the implementation of the platoon-split strategy. The formulation of the travel time savings percentage is provided below with $t_{S}$ as the time savings percentage:

$t_{S}=\frac{t_{5}-t_{4}}{t_{5}-t_{2}}$

Time $t_{5}$ can be determined from Figure 1 with the following equation:

$t_{5}=\frac{d}{w}+\frac{d}{u}$

By substitution of Equations 2, 5, and 20 into Equation 19, the following equation is determined:

$t_{S}=\left(\frac{x_{L}}{d}\right)\left(\frac{u^{-1}-v^{-1}}{u^{-1}}\right)$

From Equation 6, the following relationship is formulated:

$t_{S}=\left(\frac{w^{-1}+u^{-1}}{w^{-1}+2 u^{-1}-v^{-1}}\right)\left(\frac{u^{-1}-v^{-1}}{u^{-1}}\right)$

Equation 22 can be used to determine the theoretical time savings for the platoon-split strategy with knowledge of the various desired speeds for the EV and background traffic and for the shock wave speed.

The theoretical maximum time savings for the two-intersection scenario can be determined in a similar manner from Figure 2 by comparison of the arrival time of the EV with and without the use of the platoon-split strategy (time $t_{4}$ and time $t_{5}$, respectively). The formulation of the travel time savings percentage results in the same relationship. Therefore, the theoretical travel time savings is identical for the one-intersection and two-intersection scenarios.

Table 1 provides the theoretical travel time savings percentages for the speeds tested for the one-intersection and two-intersection scenarios. As shown in Table 1, the travel time savings percentage increases as the difference between $u$ and $v$ gets larger. 
TABLE 1 Theoretical Time Savings

\begin{tabular}{lcccc}
\hline & \multicolumn{2}{l}{ Speed $(\mathrm{k} / \mathrm{h})$} & & \\
\cline { 2 - 4 } $\begin{array}{l}\text { Speed } \\
\text { Setting }\end{array}$ & $\begin{array}{l}\text { Background } \\
\text { Traffic }(u)\end{array}$ & EV $(v)$ & $\begin{array}{l}\text { Shock Wave } \\
(w)\end{array}$ & $\begin{array}{l}\text { Travel Time } \\
\text { Savings (\%) }\end{array}$ \\
\hline 1 & 50 & 80 & 19 & 34 \\
2 & 50 & 65 & 19 & 22 \\
3 & 76 & 86 & 19 & 16 \\
\hline
\end{tabular}

\section{Simulation Results}

After each simulation run, the times when vehicles passed certain locations were recorded. The time instant when the EV joined the back of the queue (time $t_{0}$ ), the time at which the EV departed from the queue (time $t_{2}$ ), the time at which the EV changed lanes (time $t_{3}$ ), and the time at which the EV entered the upstream intersection (time $t_{4}$ ) were recorded. Simulation runs were completed both with and without the implementation of the strategy presented to compare the EV travel times and determine the travel time savings.

Figure 4 summarizes the results for each simulation run for the three speed settings for the one-intersection scenario. Figure 4 shows the percent reduction in travel time for the EV versus distance $d$. The data indicate that the time savings ranges from $3 \%$ to $35 \%$ (1 to $21 \mathrm{~s}$ ) and varies depending on the distance from the upstream intersection $(d)$. It was noticed that as $d$ increases beyond $500 \mathrm{~m}$, the increase in the travel time savings percentage begins to level off near the theoretical limit.

The percent reduction in travel times should, theoretically, not change as $d$ varies. On the basis of the desired speeds used in the simulation, the theoretical reduction in travel times should be about $34 \%$ for Speed Setting 1, about 22\% for Speed Setting 2, and 16\% for Speed Setting 3. The values are identified by the straight black lines in Figures 4 and 5. However, the LWR formulation does not account for driver reaction time or acceleration and deceleration behavior and assumes that vehicles can change speed instantaneously. When the distance $d$ is short, the driver reaction time and the acceleration and deceleration affect travel times more substantially. However, as $d$ increases, the impacts of these factors become negligible.

Figure 5 summarizes the results for the simulation runs for the two-intersection scenario with each of the three speed settings and the three intersection spacing values. Figure 5 shows the percent reduction in travel time versus the distance $d$. The solid datum points indicate that the downstream intersection queue length during the simulation run was less than the maximum queue length limitation $\left(Q_{L_{\max }}\right)$. The hollow datum points indicate that the downstream intersection queue length was greater than $Q_{L_{\max }}$.
The data for an intersection spacing of $1,000 \mathrm{~m}$ (top row) indicate that the time savings varies between $6 \%$ and $34 \%$ (4 and $26 \mathrm{~s}$ ), depending on the distance $(d)$ from the upstream intersection and the downstream queue length. It was noticed that as $d$ increases beyond $50 \mathrm{~m}$, the increase in the travel time savings percentage begins to level off near the theoretical limit. The distance $d$ at which the travel time savings approaches the theoretical value is significantly shorter (closer to the intersection) than that in the one-intersection scenario. This is due to the distance that the EV can travel at its desired speed, which is much longer for the two-intersection scenario.

The data for an intersection spacing of $500 \mathrm{~m}$ (middle row) indicate that the travel time savings varies between $0 \%$ and $33 \%$ ( 0 and $20 \mathrm{~s})$. The shape of the datum points is similar to that of the datum points for the $1,000-\mathrm{m}$ spacing, but the variability in the percentage of time saved is greater. This is because of the large number of simulation runs that had downstream queues larger than $Q_{L_{\max }}$.

The data for an intersection spacing of $250 \mathrm{~m}$ (bottom row) indicate that the time savings varies between $0 \%$ and $33 \%$ ( 0 and $11 \mathrm{~s})$. The shape of the datum points is similar to that of the datum points for the 500-m spacing, but as $d$ increases beyond $50 \mathrm{~m}$, the travel time savings does not approach the theoretical limit. This can be attributed to the fact that the downstream queue lengths are the same for each set of simulation runs. Each simulation for the scenarios with 1,000-, $500-$, and 250-m intersection spacings was run with the same random seed set, and the simulations had identical traffic patterns. The intersection spacing determines the maximum length that the downstream queue can be to provide an unimpeded path for the EV. Therefore, the number of simulation runs that have downstream queue lengths greater than $Q_{L_{\max }}$ increases when the intersection spacing decreases. If the length of the downstream queue is longer than $Q_{L_{\max }}$, the EV will have to slow down before the downstream intersection, and the slower speed will reduce the time savings percentage.

Table 2 provides the results of a statistical analysis comparing the simulation run with the $\mathrm{EV}$ with siren and the simulation run with the EV with siren and preemption, the simulation run with the EV with siren with the simulation run with the platoon-split strategy, and the simulation run with the EV with siren and preemption with the simulation run with the platoon-split strategy. The comparison includes Speed Setting 1 (background traffic traveling at $50 \mathrm{~km} / \mathrm{h}, \mathrm{EV}$ traveling at $80 \mathrm{~km} / \mathrm{h}$ ) with the two-intersection scenario. The amount of time needed to run the simulations with the EV with siren and EV with siren and preemption was large; therefore, only Speed Setting 1 with the scenario with 1,000 -m intersection spacing was performed.

The estimate indicates with $95 \%$ confidence that the difference between the EV travel times with the platoon split falls within the lower and upper intervals in Table 2. Because the value 0 is not within the intervals for any of the three comparisons, the evidence is sufficient to conclude that a difference between the EV travel

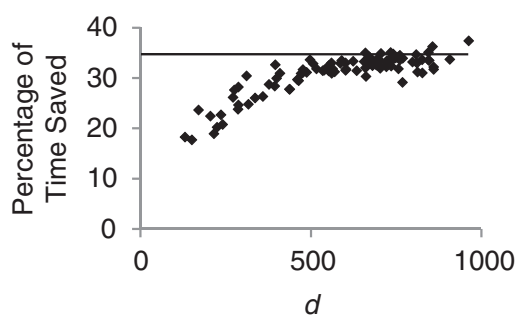

(a)

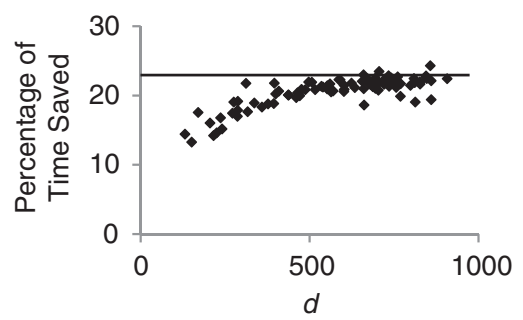

(b)

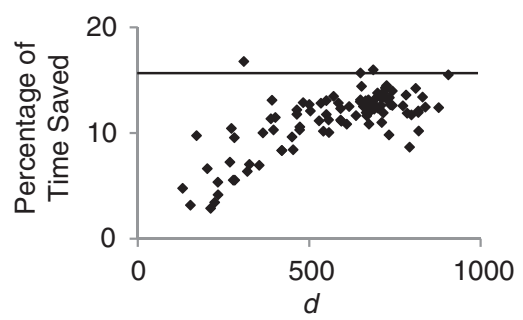

(c)

FIGURE 4 Improvement in EV travel time for one intersection at (a) Speed Setting 1, $(b)$ Speed Setting 2, and (c) Speed Setting 3. 

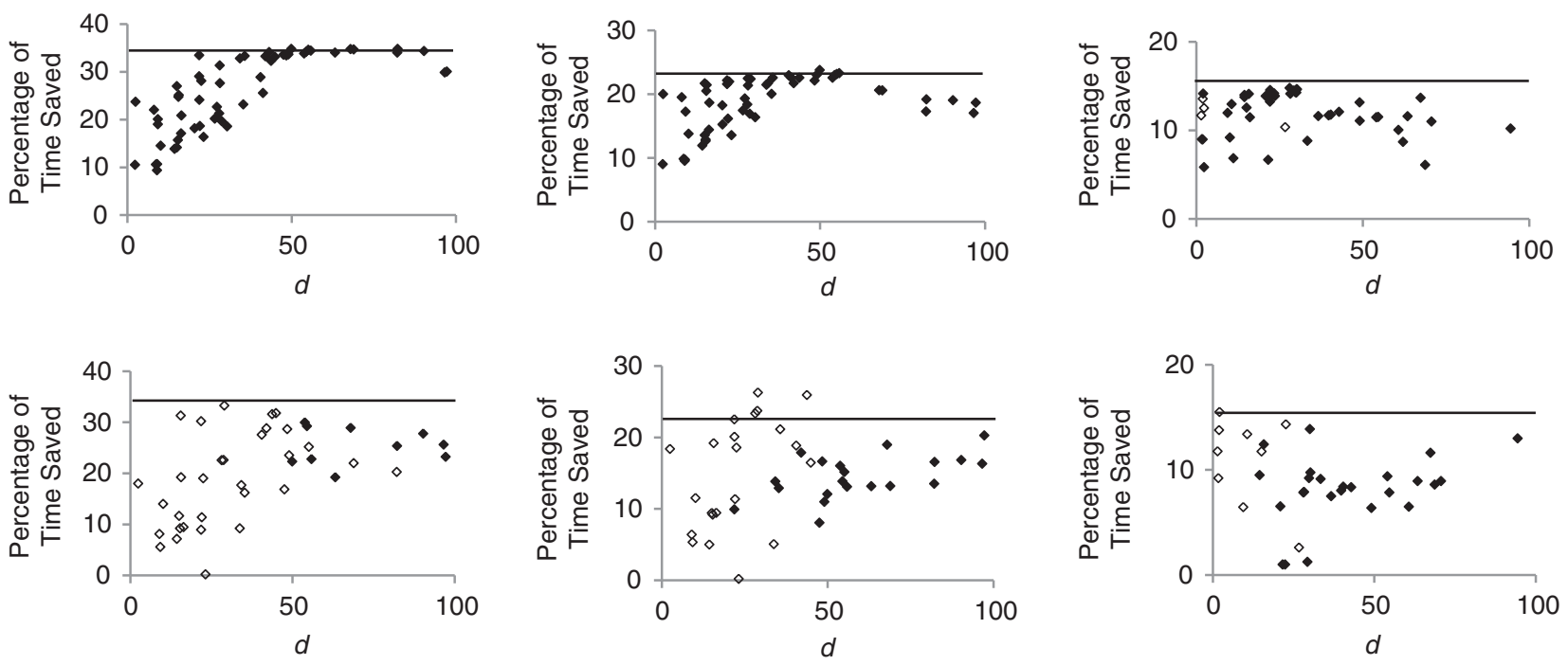

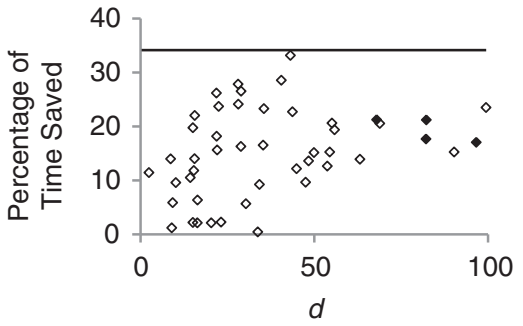

(a)

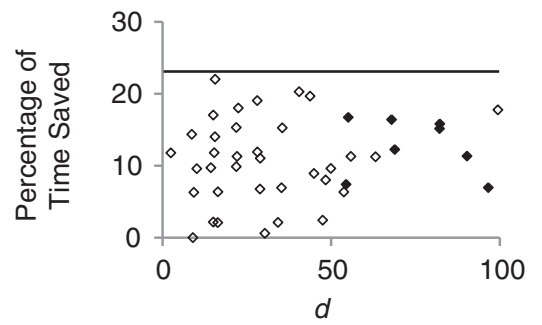

(b)

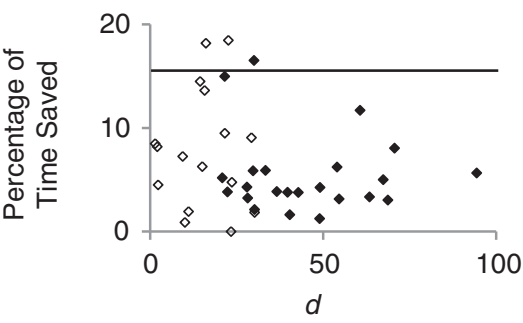

(c)

FIGURE 5 Improvement in EV travel time at different speed settings and intersection spacings: (a) Speed Setting 1, (b) Speed Setting 2, and (c) Speed Setting 3; (top row) 1,000 m, (middle row) $500 \mathrm{~m}$, and (bottom row) $250 \mathrm{~m}$.

times exists in the simulation runs with the implementation of the platoon-split strategy and those in the simulation runs with the EV with siren or the EV with siren and preemption.

\section{Market Penetration Effectiveness}

The microscopic simulation runs were performed under the assumption that the market penetration of vehicles equipped with VTV communication was $100 \%$. This will likely not occur in the foreseeable future. To evaluate the benefits of the proposed strategy at different market penetration rates, a probabilistic analysis was performed. An equation was derived for the expected travel time savings for different market penetration rates and is provided below. The expected value of the travel time savings is represented by $E$ (TTS). The formulation for the equation has been omitted because of space limitations but will be further explored in subsequent studies. The following variables were used in the formulation:

$\mathrm{TT}=$ theoretical travel time savings (identified in Table 1),

$n=$ number of vehicles in queue between intersection and critical split point $\left(x_{L}\right)$, and

$p=$ market penetration value of vehicles equipped with VTV communication.

$E(\mathrm{TTS})=\mathrm{TT}\left[\left(\frac{n+1}{n}\right)+\left(\frac{(1-p)^{n+1}-1}{n p}\right)\right]$

A graph that identifies the expected travel time savings for different market penetration rates for each of the three speed settings

TABLE 2 Average EV Travel Time Difference

\begin{tabular}{|c|c|c|c|c|c|c|}
\hline \multirow[b]{2}{*}{ Comparison } & \multirow{2}{*}{$\begin{array}{l}\text { Average Travel } \\
\text { Time Difference }(\bar{D})\end{array}$} & \multirow{2}{*}{$\begin{array}{l}\text { Standard Deviation } \\
\text { of Travel Time } \\
\text { Difference }\left(\sigma_{d}\right)\end{array}$} & \multirow[b]{2}{*}{$z$-Value $\left(z_{0.25}\right)$} & \multirow[b]{2}{*}{ Sample Size $(n)$} & \multicolumn{2}{|c|}{$\begin{array}{l}95 \% \text { Confidence } \\
\text { Interval }\end{array}$} \\
\hline & & & & & Lower & Upper \\
\hline $\begin{array}{l}\text { EV with siren versus EV with siren } \\
\text { and preemption }\end{array}$ & 8.01 & 6.48 & 1.96 & 30 & 5.69 & 10.33 \\
\hline EV with siren versus platoon split & 25.36 & 8.91 & 1.96 & 30 & 22.17 & 28.55 \\
\hline $\begin{array}{l}\text { EV with siren and preemption } \\
\text { versus platoon split }\end{array}$ & 11.36 & 5.96 & 1.96 & 30 & 9.23 & 13.49 \\
\hline
\end{tabular}




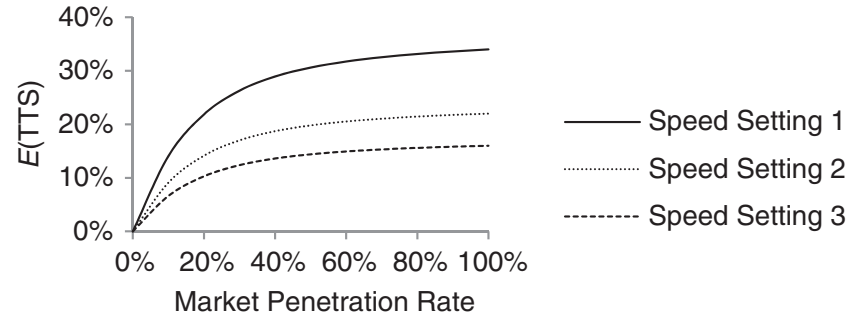

FIGURE 6 Travel time savings for different market penetration rates.

(identified in Table 1) is provided in Figure 6. The graph shows that market penetration rates greater than $20 \%$ are expected to provide travel time savings greater than $60 \%$ of the theoretical limit.

\section{CONCLUSION}

This paper evaluated a new strategy to enable EVs to traverse congested roadways and to traverse through congested intersections more quickly, an improvement that may be critical to patients' survival rates. The application of the concept was illustrated for two scenarios. The scenarios investigated splitting of the vehicle queue in one lane at a critical location so that an EV could proceed at its desired speed with minimal disruption to background traffic. The formulations were developed on the basis of the shock wave theory of traffic flow to predict the queuing behavior at signalized intersections. The proposed method was simulated in VISSIM for evaluation.

The results indicated that this strategy can significantly shorten the trip times for EVs for the one-intersection and two-intersection scenarios. The simulation results showed that travel time savings percentages approached the theoretical maximum values (ranging from $16 \%$ to $34 \%$ on the basis of the relative speeds of the EV and the other vehicles) as $d$ is increased, if a $100 \%$ market penetration rate for VTV communication is assumed. Considerable travel time savings is expected when market penetration rates are as low as $20 \%$.

Future work will expand on this research by relaxation of the controlled environment. Simulation runs will be performed with vehicle speeds randomly distributed over a larger range of values. The VTV communication system will be coded in a network simulator to simulate more accurately message propagation and will include signal degradation. Driver compliance rates will be included in the simulation runs to evaluate the effect on travel time savings. In addition, different types of vehicles other than passenger vehicles (i.e., trucks, motorcycles, and buses) will be included in the simulations. The simulation roadway network will also be expanded to include leftturning vehicles and access points between signalized intersections. Simulation runs will also be performed on larger networks with more intersections and with roadways that have more lanes.

The use of the proposed strategy will affect overall traffic performance, and some background vehicles will have increased delays. The implementation of the strategy may be dependent on the level of urgency of the emergency. The impacts of the proposed strategy will be evaluated, and trade-offs in the provision of preference to EVs will be explored.

\section{ACKNOWLEDGMENTS}

This study was funded in part by the TranLIVE University Transportation Center, a consortium of five universities funded by the U.S. Department of Transportation's Research and Innovative Tech- nology Administration. The authors thank the three anonymous reviewers for their valuable comments and suggestions.

\section{REFERENCES}

1. Park, H., C. S. Bhamidipati, and B. L. Smith. Development and Evaluation of Enhanced IntelliDrive-Enabled Lane Changing Advisory Algorithm to Address Freeway Merge Conflict. In Transportation Research Record: Journal of the Transportation Research Board, No. 2243 , Transportation Research Board of the National Academies, Washington, D.C., 2011, pp. 146-157.

2. Kato, S., S. Tsugawa, K. Tokuda, T. Matsui, and H. Fujii. Vehicle Control Algorithms for Cooperative Driving with Automated Vehicles and Intervehicle Communications. IEEE Transactions on Intelligent Transportation Systems, Vol. 3, No. 3, 2002, pp. 155-161.

3. Gupta, S. D., Y. P. Fallah, and S. E. Shladover. Sharing Vehicle and Infrastructure Intelligence for Assisted Intersection Safety. Proc., 22nd IEEE International Symposium on Personal, Indoor and Mobile Radio Communications, 2011, pp. 767-771.

4. Wenyang, G., H. Jianhua, B. Lin, and T. Zuoyin. Adaptive Congestion Control of DSRC Vehicle Networks for Collaborative Road Safety Applications. Proc., 36th IEEE Conference on Local Computer Networks, 2011, pp. 913-917.

5. Comert, G., and M. Cetin. Analytical Evaluation of the Error in Queue Length Estimation at Traffic Signals from Probe Vehicle Data. IEEE Transactions on Intelligent Transportation Systems, Vol. 12, 2011 pp. 563-573.

6. Comert, G., and M. Cetin. Queue Length Estimation from Probe Vehicle Location and the Impacts of Sample Size. European Journal of Operational Research, Vol. 197, 2009, pp. 196-202.

7. Xu, H., and M. J. Barth. Travel Time Estimation Techniques for Traffic Information Systems Based on Intervehicle Communications. In Transportation Research Record: Journal of the Transportation Research Board, No. 1944, Transportation Research Board of the National Academies, Washington, D.C., 2006, pp. 72-81.

8. Buchenscheit, A., F. Schaub, F. Kargl, and M. Weber. A Vanet-Based Emergency Vehicle Warning System. Proc., 2009 IEEE Vehicular Networking Conference, 2009.

9. Richards, P. I. Shock Waves on the Highway. Operations Research, Vol. 4, No. 1, 1956, pp. 42-51.

10. Caelli, T., and D. Porter. On Difficulties in Localizing Ambulance Sirens. Human Factors, Vol. 22, No. 6, 1980, pp. 719-724.

11. Auerbach, P. S., J. A. Morris, Jr., J. B. Phillips, S. R. Redlinger, and W. K. Vaughn. An Analysis of Ambulance Accidents in Tennessee. The Journal of the American Medical Association, Vol. 258, No. 11, 1987, pp. $1487-1490$.

12. Grant, C. C., and B. Merrifield. Analysis of Ambulance Crash Data. The Fire Protection Research Foundation, Quincy, Mass., 2011.

13. Custalow, C. B., and C. S. Gravitz. Emergency Medical Vehicle Collisions and Potential for Preventive Intervention. Prehospital Emergency Care, Vol. 8, No. 2, 2004, pp. 175-184.

14. Pell, J. P., J. M. Sirel, A. K. Marsden, I. Ford, and S. M. Cobbe. Effect of Reducing Ambulance Response Times on Deaths from Out of Hospital Cardiac Arrest: Cohort Study. British Medical Journal, Vol. 322 No. 7299, 2001, pp. 1385-1388.

15. ECC Committee, Subcommittees and Task Forces of the American Heart Association. 2005 American Heart Association Guidelines for Cardiopulmonary Resuscitation and Emergency Cardiovascular Care. Circulation, Vol. 112, Supplement 24, 2005, pp. IV-1-IV-5.

16. Moussa, N. Evacuation Model for Emergency Vehicles in Highways. International Journal of Modern Physics, Vol. 20, No. 1, 2009, pp. 59-69.

17. Toy, C., K. Leung, L. Alvarez, and R. Horowitz. Emergency Vehicle Maneuvers and Control Laws for Automated Highway Systems. Proc. IEEE Transactions on Intelligent Transportation Systems, Vol. 3, No. 2 , 2002, pp. 109-119.

18. Yoo, J. B., J. Kim, and C. Y. Park. Road Reservation for Fast and Safe Emergency Vehicle Response Using Ubiquitous Sensor Network. Proc. IEEE International Conference on Sensor Networks, Ubiquitous, and Trustworthy Computing, 2010, pp. 353-358.

19. Liu, H. X., X. Wu, W. Ma, and H. Hu. Real-Time Queue Length Estimation for Congested Signalized Intersections. Transportation Research Part C, Vol. 17, No. 4, 2009, pp. 412-427.

The Intelligent Transportation Systems Committee peer-reviewed this paper. 